\title{
Social Referencing and the Security of Attachment*
}

\author{
SUSAN DICKSTEIN \\ University of Illinois \\ ROSS A. THOMPSON \\ University of Nebraska \\ DAVID ESTES \\ University of Michigan
}

\author{
CATHERINe MALKIN AND Michael E. LAMB \\ University of Utah
}

\begin{abstract}
The second episode of the Strange Situation procedure was used to study individual differences in infants' social referencing and relationships to the security of attachment. This 3-min episode entails interaction with an unfamiliar adult in which infants may seek emotional cues from mother. Forty-three $191 / 2$-month-old infants were observed. From videotaped records, the security of attachment was assessed using Ainsworth's criteria. For episode 2, minute-by-minute rotings of infont referencing and proximity to mother, and the quality of maternal utterances (to assess maternal emotional cuing) were performed. Referencing declined throughout the episode and was negatively related to proximity. A marginally significant $(p<.06)$ main effect for attachment classification revealed that insecureresistant infants referenced most frequently (and showed persistent referencing early in the episode), insecure-ovoidant babies referenced least frequently, with securely attached infants in between. Mothers displayed a positive vocal tone to the baby but did not vary with infant attachment status. These findings suggest that referencing may reflect aspects of secure base behavior which distinguish securely from insecurely attached infants.
\end{abstract}

social referencing security of attachment secure base behavior socioemotional development

mother-infant interaction

Recent research on early emotional development has focused on the social origins and functions of emotional signals. Behaviors like crying, smiling, and cooing are potent social signals, and their occurrence is often affected by the baby's perception of emotional cues from others. This phenomenon-known

" This study is based on an undergraduate Senior Honors' Thesis conducted by the first author at the University of Michigan. The research was supported by a grant from the Riksbankens Jubileumsfond of Sweden to M. Lamb and by a dissertation grant from the Rackham School of Graduate Studies to R. Thompson. We are grateful for the assistance provided by Susan Piconke, Jamie Steinberg, and Susan Strzelecki, and for technical advice from Lec Davis. Finally, we wish to express a special debt to the families who participated in this study. An earlier version of this paper was presented to the biennial meeting of the International Conference on Infant Studies, Austin, Texas, March 1982. Requests for reprints and correspondence should be sent to Susan Dickstein, Department of Psychology, University of Ilinois, Champaign, IL 61820. 
as "social referencing" - has been studied developmentally and in various contexts. Social referencing may be defined as actively seeking cues from others in an uncertain situation and using these cues to facilitate appraisal of the event (Campos \& Stenberg, 1981; Klinnert, Campos, Sorce, Emde, \& Svejda, 1983). The primary purpose of this study was to examine individual differences in social referencing among infants and their relation to the security of attachment.

With infants and young children, of course, the mother and other significant caregivers are likely to be the targets of social referencing. Research to date has shown that (a) infants as young as 7 months old engage in maternal referencing (Sorce, Emde, \& Frank, 1982), (b) infants make active efforts to stay within view of their mothers' faces in unfamiliar settings (Carr, Dabbs, \& Carr, 1975), and (c) maternal unresponsiveness inhibits the baby's exploratory activity (Sorce \& Emde, 1981). Further, babies will play with novel, unusual toys and cross the deep side of the visual-cliff when their mothers are smiling, but will not do so when their mothers look frightened (Klinnert, 1981; Sorce, Emde, \& Klinnert, 1981; Svejda, 1981). Thus infants reliably seek and respond appropriately to their mothers' emotional cues before the end of the first year.

Thus far, few researchers have examined individual differences in maternal referencing, although these differences may reflect an important aspect of the mother-infant relationship. It is likely that the extent to which infants seek emotional cues from their mothers in uncertain circumstances may depend on their past success in obtaining such assistance. In this respect, differences in maternal referencing may be related to the variations in secure base behavior which apear to differentiate securely attached from insecurely attached infants as'reflected by their Strange Situation behavior (Ainsworth, Blehar, Waters, \& Wall, 1978). Securely attached infants would thus be likely to reference their mothers when faced with uncertainty, and decrease the frequency of their referencing as they become more comfortable with the situation. In contrast, insecurely attached infants may either (a) reference little in novel, uncertain situations, or (b) reference persistently because of their continued uncertainty with the situation.

In order to explore the relationships between the security of attachment and maternal referencing, we studied referencing in the second episode of the Strange Situation. During this 3-min episode, the infant encounters an unfamiliar female adult who enters the room and sits silently, then talks to the mother and later begins to play with the infant. Uncertainty about the stranger should lead infants to seek emotional cues from their mothers. Since behavior during episode 2 does not figure prominently in appraising the security of attachment, there was little overlap between our assessments of maternal referencing and attachment security. From videotaped records, we calculated the frequency of maternal referencing for each minute, as well as recording the infant's proximity to the mother. In addition, we assessed the kinds of emotional cues provided by.the mother by rating the quality of maternal vocalizations on 
a minute-by-minute basis. Maternal vocalizations were chosen because they are a source of emotional information upon which infants can rely without even having to look to the mother, and because they probably constitute a more sensitive index of emotional cues than facial expressions do (see Ekman, Friesen, O'Sullivan, \& Scherer, 1980; Scherer, Koivumaki, \& Rosenthal, 1972).

Thus the goals of this study were to (a) examine changes in the frequency of maternal referencing over the 3 min of this episode, (b) explore individual differences in referencing and their relationships to Strange Situation behavior, (c) examine the quality of maternal vocal cues to the baby throughout the episode, and (d) study the associations between referencing and the baby's distance from the mother and the quality of maternal vocal cues.

\section{METHOD}

\section{Subjects}

Forty-three infants (21 males; 15 firstborn) were observed between their 19month and 20-month birthdays.' Families were contacted either through a research pool or through published birth announcements. Roughly $78 \%$ of the families we contacted agreed to participate. According to scores on Hollingshead's (1975) Four-Factor Index of Social Status, this was a relatively heterogenous middle-class sample. Fourteen families were in Hollingshead's Class I (major professional and business), 12 in Class II (minor professional and technical), 13 in Class III (skilled craftsmen, clerical, sales workers), and 4 in Class IV (machine operators and semiskilled workers). All families except one were intact, two-parent families.

\section{Procedure}

Infants and mothers were observed in the Strange Situation procedure (Ainsworth et al., 1978), a semistandardized procedure for assessing the security of infant-parent attachment relationships. ${ }^{2}$ The procedure consists of seven 3-min episodes designed to create gradually escalating stress for the baby (i.e., unfamiliar room, interaction with a strange adult, separation from mother in the company of that adult, and finally being left alone), so that changes in the organization of the infant's behavior in relation to the parent can be observed. In appraising the security of attachment, particular attention is devoted to the infant's behavior upon reunion following brief separations from the mother (see Ainsworth et al., 1978, for further details).

Maternal referencing was assessed in episode 2 of the Strange Situation, when an unfamiliar female adult joined mother and baby in the playroom. For the first minute of this episode, the stranger sat quietly and spoke to neither infant nor mother. During the second minute the stranger conversed with day.

'There was one exception: One infant was observed 1 week following her 20-month birth-

\footnotetext{
2 The Strange Situation was preceded by a brief (5-min) assessment of stranger sociability.
} 
mother but did not engage the baby. Finally, in the third minute the stranger moved to the floor and played with the baby. Thus as the episode proceeded, the stranger behaved in an increasingly friendly manner.

The assessment took place in a carpeted laboratory playroom containing two chairs (for mother and stranger), a table, attractive wall hangings, and an assortment of toys in the middle of the room. Videotaped observations were conducted from behind one-way windows. Five female undergraduate students served as strangers.

\section{Measures}

The security of attachment was assessed using Ainsworth's interactive variables (scored on an episode-by-episode basis) and her criteria for classification of infants into groups and subgroups based on the infant's tendency to use the adult as a secure base for exploration and ability to reunite positively after brief separations (see Ainsworth et al., 1978). Securely attached (group B) infants play comfortably in their parents' presence and greet or seek contact with them upon reunion. Insecure-avoidant (group A) babies play noninteractively during the first episode, and avoid or ignore parents during reunion episodes. Insecure-resistant (group C) babies do not play comfortably during preseparation, and upon reunion mingle contact-seeking behaviors with angry, resistant behaviors.

Videotapes of the Strange Situation sessions were scored by two or more undergraduate research assistants. Exact agreement on overall attachment classifications between independent raters was $98 \%$. The one disagreement was resolved by discussion.

The frequency of maternal referencing per minute in episode 2 was the number of times the baby looked to mother's face directly after looking to the stranger. Proximity to mother was defined for each minute by the distance between infant and mother for the majority of the period. A 3-point scale was used to distinguish (1) periods in which mother and baby were further than $2 \mathrm{ft}$ apart, (2) periods in which infant and mother were less than $2 \mathrm{ft}$ apart, and (3) periods in which mother and baby were in physical contact.

Other measures focused on maternal behavior. First, the number of maternal utterances was calculated for each minute of episode 2 . An utterance was defined as a vocal phrase preceded and followed by 2-s pauses or clear-cut changes in vocal inflection (Schaffer \& Crook, 1980). Second, the vocal tone of each utterance was rated on a 7-point scale, with a mean score for maternal vocal tone later calculated for each minute of episode 2 . A rating of 7 was assigned when maternal tone was extremely positive (characterized by a high pitch, soft intensity, and an animated, variable sound quality), while a score of 1 reflected an extremely negative tone (characterized by a low pitch, hard or harsh intensity, with unvarying sound quality). A neutral tone was flat or monotonic, and defined the midpoint of the scale. Third, a transcript of the episode was used to classify the semantic quality of each maternal utterance 
using seven summary categories: commands, instructions, questions, positive affective expressions, negative affective expressions, explanations, and "moodless" utterances. The frequency of each type of utterance was calculated for each minute of the episode.

These ratings were performed by undergraduate research assistants who obtained a high level of interrater agreement during training. An independent rescoring of eight sessions by a second rater yielded exact agreement of $\mathbf{8 7 \%}$ for maternal referencing, and $96 \%$ for infant proximity. Transcripts for eight sessions were independently rescored by a second rater to yield $95 \%$ exact agreement for the mother's semantic quality. Seven sessions (totalling 254 maternal utterances) were rescored to yield $88 \%$ exact agreement for maternal vocal tone. Ratings of infant and maternal behaviors during episode 2 were performed independently by raters who had no knowledge of the baby's attachment classification.

All statistical analyses in the following section used two-tailed tests of significance.

\section{RESULTS}

\section{Normative Trends}

For the sample as a whole, the frequency of maternal referencing declined over successive minutes of episode $2\left(M_{1}=2.47, M_{2}=1.98, M_{3}=0.49\right)$. Differences between the first and third minutes were statistically significant, $t(41)=5.78$, $p<.001$, as were differences between the second and third minutes, $t(41)=$ $4.13, p<.001$. Infants who referenced frequently during the first minute were also more likely to reference during the second minute, $r(41)=.55, p<.0005$, indicating some stability in individual differences. This association was not apparent during the second and third minutes of episode 2 , however, $r(41)=.04$, perhaps because the frequency of referencing was low during the last minute. Infants established closer proximity to mother during the second minute of episode 2 than during the first minute $\left(\mathrm{M}_{1}=1.91, \mathrm{M}_{2}=2.21\right), t(41)=-3.31$, $p<.002$; then they moved away somewhat during the final minute $\left(\mathbf{M}_{3}=1.88\right)$, $t(41)=2.74, p<.009$. Individual differences in proximity seeking were highly stable from the first to the second minute, $r(41)=.72, p<.0001$, and from the second to the third minute, $r(41)=.53, p<.0005$. There was a negative correlation between referencing and proximity during the first minute of the episode, $r(41)=-.44, p<.005$, indicating that infants who referenced the most were those who were furthest away. Similar, but nonsignificant, trends were evident in minute $2, r(41)=-.25, p<.11$, and minute $3, r(41)=-.26, p<.10$ (significance might have been achieved with a somewhat more sensitive measure of proximity).

As expected, the number of maternal utterances more than doubled in the second minute as the mothers talked to the stranger $\left(M_{1}=3.88, M_{2}=11.32\right)$, $t(41)=9.82, p<.0001$. Frequency of utterances declined during the third min- 
ute $\left(\mathrm{M}_{3}=5.09, t(41)=-7.40, p<.0001\right.$. Individual differences in the frequency of maternal utterances were stable for minutes 1 and $2, r(41)=.32, p<$ .02 , but not for minutes 2 and 3 of the episode, $r(4 !)=.17$, n.s. The mother's vocal tone also changed significantly over time, with a somewhat positive tone apparent during the first minute $\left(M_{1}=4.77\right)$, a more neutral tone during the second minute $\left(\mathrm{M}_{2}=4.37\right), t(41)=4.56, p<.0005$, and a more positive tone apparent for the final minute $\left(\mathrm{M}_{3}=4.67\right), t(41)=-2.39, p<.025$. Individual differences in maternal tone were also consistent from the first to second minutes, $r(41)=.39, p<.01$, and second to third minutes, $r(41)=.37, p<.05$. Maternal utterances thus seemed more likely to have a positive tone when directed to the baby (minutes 1 and 3 ) than when mother was conversing with the stranger (minute 2). There were, however, no significant correlations between frequency of maternal utterances and infant referencing, nor between maternal vocal tone and infant referencing, for any minute of the episode.

Finally, a multivariate anaiysis of variance was conducted to determine the effects of minutes in episode 2 as well as the baby's attachment classification on the seven semantic categories of maternal vocalizations. The MANOVA revealed an overall significant effect for minutes, multivariate $F(14,27)=21.33$, $p<.0001$, which was attributable to a decrease in maternal instructions, $F(2,39)$ $=9.19, p<.0005$, and questions, $F(2,39)=9.95, p<.0003$, as the episode proceeded, while explanations, $F(2,39)=54.03, p<.0001$, and "moodless" utterances, $F(2,39)=29.17, p<.0001$, first increased during minute 2 , then decreased in minute 3 . The remaining results are discussed in the next section.

\section{Associations with the Security of Attachment}

The distribution of infants across attachment classifications accorded with the normative trends reported by Ainsworth et al. (1978): 67\% $(n=29)$ were classified as securely attached, $14 \%(n=6)$ were rated insecure-avoidant, and $19 \%$ $(n=8)$ were judged insecure-resistant. Throughout episode 2, resistant infants referenced most frequently, avoidant infants least frequently, with securely attached babies between these two groups (see Fig. 1). A two-way (Minutes x Attachment) repeated-measures ANOVA revealed a marginally significant main effect for attachment classification, $F(2,40)=3.03, p<.06$, as well as a highly significant main effect for minutes, $F(2,80)=17.01, p<.001$, confirming the pattern earlier described. There was no significant interaction between these factors. ${ }^{3}$ Inspection of Figure 1 reveals that the most striking differences appeared during the second minute of episode 2 , when resistant infants actually referenced more while infants in the other $t$ wo groups referenced less. In pair-

3 We also partitioned the secure classification into subgroup clusters in order to examine whether there was significant heterogeneity within the secure group in maternal referencing (cf. Thompson \& Lamb, 1983). The resulting ANOVA using this four-group comparison (i.e., A vs. $B_{1,2}$ vs. $B_{3,4}$ vs. $\left.C\right)$ yidded a nonsignificant main effect for attachment group, $F(3,39)=2.66$, n.s., although the main effect for minutes remained significant, $F(2,78)=17.67, p<.001$. There was no significant interaction between these factors. In pairwise comparisons, there were no significant differences between $B_{1,2}$ and $B_{3,4}$ clusters for any minute of episode 2 . In short, dividing the secure classification into subgroups clusters did not add significantly to explained variance in referencing. 


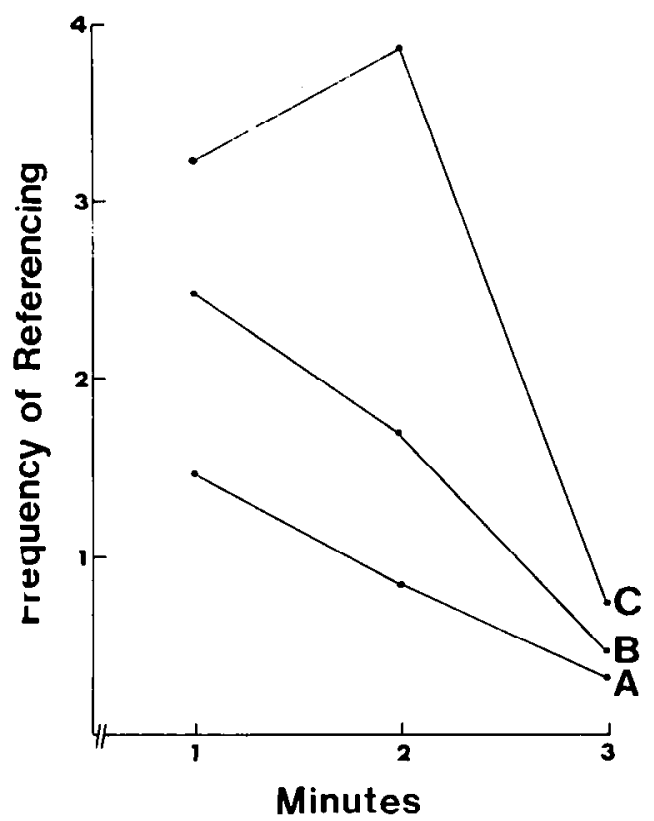

Figure 1. Frequency of social referencing as a function of (a) minutes of Strange Situation episade 2, and (b) infant attachment classification (insecure-avoidant $[A]$, secure $[B]$, or insecure-resistant $[C]$ ).

wise comparisons between A, B, and C groups for each minute of the episode, significant differences were apparent between groups $B$ and $C$ for the second minute, $t(35)=2.27, p<.05$, and between groups $\mathrm{A}$ and $\mathrm{C}$ for the same minute, $t(12)=2.57, p<.05$. There were no other significant differences between attachment classifications in their proximity to mother.

Another two-way repeated-measures ANOVA for maternal tone revealed that the mothers of infants in the three attachment classifications did not differ significantly in vocal tone; the main effect for minutes was significant, $F(2,80)=7.07, p<.01$, as earlier described. There was no interaction between these factors. Another two-way repeated-measures ANOVA was performed to assess differences in the frequency of maternal utterances. This revealed significant differences for the baby's attachment classification, $F(2,40)=4.06, p<$ .025 , with mothers of avoidant babies $(M=9.78)$ talking more than the mothers of securely attached infants $(M=6.38)$ and the mothers of resistant babies $(M=5.92)$. There was also a main effect for minutes of episode 2, $F(2,80)=50.57, p<.0001$, to confirm the pattern earlier described, but no interaction between these factors.

Finally, results of the MANOVA for maternal semantic categories described earlier revealed a significant effect for infant attachment classification, multivariate $F(14,68)=1.91, p<.04$, which was attributable to differences in maternal commands, $F(2,40)=3.37, p<.05$ (mothers of $\mathrm{A}$ babies $[\mathrm{M}=2.33]$ used more commands than did mothers of $B$ infants $[M=1.17]$ and $C$-group 
mothers $[\mathrm{M}=1.13]$ ), and instructions, $F(2,40)=6.96, p<.0025$ (C mothers $[M=0.46]>A$ mothers $[M=0.39]>B$ mothers $[M=0.08]$ ). There was also a significant interaction between minutes and attachment classifications, multivariate $F(28,54)=1.74, p<.05$, which was attributable to an increase in instructions by $C$-group mothers during the second minute of the episode, $F(4,78)$ $=5.65, p<.0005$.

\section{DISCUSSION}

These analyses revealed important characteristics of both infant and maternal behavior during the 3-min episode we studied. First, infants referenced most frequently directly following the stranger's arrival, and referencing declined at the end of the episode, suggesting that referencing was affected by uncertainty about the unfamiliar adult. Second, proximity to mother followed a different course: Infants first moved toward their mothers, and then moved back away from them as the episode progressed. Proximity-seeking is, of course, another adaptive response to uncertainty, and the decrease in proximity from the second to third minutes-like the decline in referencing-may reflect the infants' reduced uncertainty about the situation. Third, there was a negative association between referencing and proximity: Infants who were closest to mother referenced the least. This may occur because proximity-seeking and referencing are alternative strategies for checking back with mothers in uncertain situations: Infants may either use a distal mode (i.e., referencing) or a more proximal mode (i.e., approach) for reassurance (cf. Thompson \& Lamb, 1983).

Maternal behavior also changed as the episode progressed. In particular, the mother's vocal tone was most positive when she was talking to the baby (i.e., minutes 1 and 3 ) and most neutral (or businesslike) when conversing with the stranger (i.e., minute 2 ). Thus infants generally received reasonably positive cues from their mothers. Interestingly, the infants moved closer to their mothers during the period when maternal tone was most negative, and moved away from her during the period when vocal tone became positive again. Changes in infant proximity may also have been due to the mother's inattentiveness when conversing with the stranger (cf. Sorce \& Emde, 1981). Finally, the semantic quality of maternal vocalizations also changed, with instructions and questions declining in frequency as the episode proceeded, and explanations and "moodless" utterances increasing when mothers conversed with the stranger. Thus the content of maternal utterances changed as the episode proceeded, even though their vocal tone to the baby remained positive.

In addition to these normative trends, individual differences in infant behaviors were also discerned. First, individual differences in infant (and maternal) behaviors were generally stable throughout this episode, especially during the first $2 \mathrm{~min}$. Second, throughout the episode, resistant (group C) babies referenced the most, avoidant (group A) infants referenced the least, with securely attached (group B) infants between these groups, although these 
differences were marginally significant. The most striking differences appeared in the second minute when resistant babies referenced more and the other infants referenced less than in the first minute. This heightened and persistant referencing may derive, in fact, from the general anxiety and uncertainty of the $C$ babies noted by Ainsworth and Wittig (1969), combined with their inability to use the mother as a source of quick reassurance. The avoidant infants, in contrast, referenced least of all, reflecting a general tendency to ignore their attachment figures (even in uncertain circumstances) and perhaps also their lesser wariness of the stranger. Thus the individual differences in maternal referencing during episode 2 are consistent with the patterns of mother-directed behavior exhibited by these infants during other episodes of the Strange Situation, which may reflect certain aspects of secure base behavior which appear to distinguish securely from insecurely attached infants. These findings are important in light of recent controversy regarding the interpretation of Strange Situation behavior (Lamb, Thompson, Gardner, Charnov, \& Estes, 1984), and suggest that "security of attachment" as assessed within the Strange Situation is meaningfully related to other aspects of infant socioemotional behavior.

Variations in maternal behavior, however, presented an inconsistent picture. On one hand, the mothers of avoidant infants generally talked more and used more commands than did other mothers, while the mothers of resistant infants provided more instructions. On the other hand, there were no reliable differences in the vocal tone of mothers whose infants differed in attachment security. Thus variations in the content of maternal utterances were not reflected in the tone in which they were conveyed. Since tone is likely to be the more important source of emotional cuing for infants at this age, these findings suggest that the group differences in referencing between secure, avoidant, and resistant infants were not entirely attributable to variations in mothers' immediate cues. Rather, they may have derived, in part, from infant expectations resulting from a history of prior situations requiring social referencing.

While these findings suggest that there is a relationship between the security of attachment and maternal referencing in the Strange Situation, they should be interpreted cautiously in light of the marginal significance of the $\mathrm{A}$, B, C group differences in referencing, and the fact that differences were most apparent during the second minute of the episode. In addition, our results are different from those of Bradshaw, Goldsmith, and Campos (1984), who found few relationships between infants' social interactive behaviors in the Strange Situation and independent measures of social referencing (attachment classifications were not used). In their study, scores on an infant temperament measure were more strongly related to the referencing measures than to the attachment measures. Taken together, these studies suggest that multiple influences are likely to contribute to the emergence of individual differences in social referencing. Further exploration of these influences is likely to yield insights into this important socioemotional phenomenon. 


\section{REFERENCES}

Ainsworth, M. D. S., Blehar, M. C., Waters, E., \& Wall, S. (1978). Patterns of attachment. Hillsdale, NJ: Erlbaum.

Ainsworth, M. D. S., \& Wittig, B. A. (1969). Attachment and exploratory behavior of one-yearolds in a strange situation. In B. M. Foss (Ed.), Determinants of infant behaviour IV. London: Methuen.

Bradshaw, D. L., Goldsmith, H. H., \& Campos, J. J. (1984). Attachment, temperament, and social referencing: Interrelationships among three domains of infant affective behavior. Unpublished manuscript, University of Denver, Denver, CO.

Campos, J. J., \& Stenberg, C. R. (1981). Perception, appraisal and emotion: The onset of social referencing. In M. E. Lamb \& L. R. Sherrod (Eds.), Infant social cognition. Hillsdale, NJ: Erlbaum.

Carr, S., Dabbs, J., \& Carr, T. (1975). Mother-infant attachment: The importance of the mother's visual field. Child Development, 46, 331-338.

Ekman, P., Friesen, W. V., O'Sullivan, M., \& Scherer, K. (1980). Relative importance of face, body, and speech in judgments of personality and affect. Journal of Personality and Social Psychology, 38, 270-277.

Hollingshead, A. B. (1975). Four factor index of social status. Unpublished manuscript, Yale University, New Haven, CT.

Klimert, M. (1981, April). Infants use of mothers' facial expressions for regulating their own behavior. Paper presented at the meeting of the Society for Research in Child Development, Boston, MA.

Klinnert, M. D., Campos, J. J., Sorce, J. F., Emde, R. N., \& Svejda, M. (1983). Emotions as behavior regulators: Social referencing in infancy. In R. Plutchik \& H. Kellerman (Eds.), Emotion: Theory, research and experience. Vol. 2. Emotions in early development. New York: Academic.

Lamb, M. E., Thompson, R. A., Gardner, W., Charnov, E. L., \& Estes, D. (1984). Security of infantile attachment as assessed in the "Strange Situation": Its study and biological interpretation. Behavioral and Brain Sciences, 7, 127-144.

Schaffer, H. R., \& Crook, C. K. (1980). Child compliance and maternal control techniques. Development Psychology, 16, 54-61.

Scherer, K. R., Koivumaki, J., \& Rosenthal, R. (1972). Minimal cues in the vocal communication of affect: Judging emotions from content-masked speech. Journal of Psycholinguistic Research, 1, 269-285.

Sorce, J. F., \& Emde, R. N. (1981). Mother's presence is not enough: Effect of emotional availability on infant exploration. Developmental Psychology, 17, 737-745.

Sorce, J. F., Emde, R. N., \& Frank, M. (1982). Maternal referencing in normal and Down's syndrome infants: A longitudinal analysis. In R. N. Emde \& R. Harmon (Eds.), The development of attachment and affiliative systems. New York: Plenum.

Sorce, J. R., Emde, R. N., \& Klinnert, M. (1981, April). Maternal emotional signaling: Its effects on the visual cliff behavior of one-year-olds. Paper presented at the meeting of the Society for Research in Child Development, Boston, MA.

Svejda, M. J. (1981). The development of infant sensitivity to affective messages in the mother's voice (Doctoral dissertation, University of Denver). Dissertation Abstracts International, 42, 4623B.

Thompson, R. A., \& Lamb, M. E. (1983). Security of attachment and stranger sociability in infancy. Developmental Psychology, 19, 184-191. 